\title{
CE QUE LE JEUNE JURISTE PEUT APPORTER AUX ORGANISATIONS INTERNETIONALES '
}

\author{
(Un résumé) \\ Prof. Bülent Nuri ESEN
}

1.- Il peut paraître contradictoire de se demander ce que le jeune juriste peut apporter aux organisations internationales, tandis qu'il se trouve, vu sa situation sociale, en état non pas de donner, mais plutôt de recevoir. Pourtant, le jeune juriste peut et doit apporter bien des choses aux organisations internationales.

2.- Le cadre et le domaine d'agir de de ces genres d'organisations sont agencés par le juriste. Il peut y avoir progrès si le juriste est jeune. Le jeune juriste n'est pas toujours celui qui n'a pas atteint encore un certain âge. C'est celui qui prospecte l'avenir, qui va de l'avant, qui crée toujours le mieux. Dans ce sens, un CASISIN est perpétuellement jeune, tandis qu'un nouvel insorit fanatique et superstitieux du barreau est bien vieux malgré son jeune âge. Le jeune juriste c'est donc celui qui pense jeune. Il possède la raison critique, il aime la vie, il rêve d'un avenir meilleur, il croit en l'homme, il croit en la paix internationale.

3.- Une remarque s'impose. La vie des entités étatiques est, à l'époque dans laquelle nous vivons, intimement liée aux rapports internationaux. L'Etat envoie ses ramifications dans le domaine international. On peut voir sa projection dans la sphère des relations supra nationales. Il est, par conséquent, évident que le système international, basé sur des normes juridiques, se trouve en devenir continuel, soit par les efforts du monde international, soit par ceux des Etats qui en font partie.

Les Etats ne sont plus depuis longtemps des secteurs fermés dans lesquels les nationaux déploient leurs activités aussi bien

\footnotetext{
${ }^{1}$ Bangkok Konferansı için istenen tebliğin özetidir.
} 
qu'ils exposent leurs mérites. On sait qu'à l'issue de la Seconde Guerre Mondiale la conception de l'Etat a subi des modifications. L'Etat social, qui avait déjà fait son apparition dès la fin de 1917, s'institutiornalisa. On vit dans les constitutions d'après 1945 des dispositions qui mettaient l'Etat dans la situation d'un prestateur envers le citoyen. Ce fut l'avènement de «l'Etat Débiteur».

4.- Parallèlement à cette nouvelle conception, l'homme devint une valeur universelle sans égal. Et tout ce qui l'intéressait commençait à être considéré comme la chose de tout être humain. $\mathrm{Ce}$ qui fit que l'individu fit son apparition sur la scène internationale. Il est donc tout à fait naturel que le jeune juriste vienne voir de près ce qui se passe dans la vie internationale.

5.- Le jeune juriste est au premier abord intéressé par lcs questions qui touchent le droit. Sa qualité de jeune fait qu'il apporte un scin particulier aux promlèmes juridiques de la jeunes. se. Il connaît assez bien ces problèmes. Ce sont les siens. Il ne pourra probablement pas suggérer des solutions définitives. Mais, il posera les problèmes sous leur véritable aspect. Et cela permettra à céux qui portent la responsabilité de gouverner, de prendre des décisions convenables.

6.-- Le droit n'étant plus un amas de normes comme on le considérait jadis mais un système dynamique indiquant en même temps les moyens d'action dirigé vers l'avenir, c'est de lui qu'on coit se servir pour préparer et modeler la société future. Le droit engendrera l'idéal que la communauté humaine entend atteindre. Il s'agit de réaliser une oeuvre d'avenir. Le jeune juriste peut et doit être un des principaux artisans de cette ceuvre. Il contribuera à bâtir le lerdemain. Il devra, par conséquent, chercher le moins dommageable, sinon le perfectionné.

7.- Les expériences séculaires ont montré combien lents et ingrats furent les efferts pour construire un monde meilleur. Il a fallu préparer les idées. Il a fallu convaincre. Il a fallu obtenir l'action cormune. Si des résultats, hélas facilement vulnérables ont été atteints, c'est encore grâce à une compréhension entre les peup. les.

8.- Il arrive que le sens même d'une compréhension disparaisse, que la raison soit détruite par ceux que l'on croyait les plus raisonnables. La société politique "classique» a eu, jusqu'à présent, st's propres règles. Elle a plongé dans un conflit international. Et les dirigeants ont décidé du sort des masses adolescentes. 
Or, ce à quoi nous assistons de nos jours et ce qu'on a eu l'habitude d'appeler «la contestation étudiante» a une signification particulièrement importante. Tous les jeunes, de tous les pays font preuve des mêmes conceptions. L'ordre existant ne leur plaît pas. Ils veulent un monde différent. Un monde qui aura ses propres règles de vie. Ce sera sîrement un nouveau cadre juridique, différent du nôtre. Les artisans de ce cadre futur seront les jeunes juristes. Ils construiront la future société humaine. Certes, ils n'iront pas jusqu'à inventer tout, de nouveau. Mais, ils assureront la transition à un système meilleur. Pour cela ils découvriront des nouvelles normes aptes à gouverner une société améliorée. Les dirigeants actuels du monde se trouvent divisés. Les jeunes ne le sont pas. Ils ont la conscience d'être de la même pâte. Tandis que certains pays, soucieux de leur avenir et désireux de s'assurer au moins une sécurité régionale désirent la formation de règles de droit international distinct - ce qui ruine le respect au droit international tout court - les générations des jeunes juristes sont pour la création d'un monde meilleur basé sur des principes juridiques et des valeurs morales communs.

9.- Il s'agirait de l'unification des principes essentiels dans les différents systèmes juridiques. C'est de là que naîtrait le vrai rapprochement entre les peuples. L'U.N.E.S.C.O. s'est attaché et tient encore à découvrir et faire admettre les valeurs de culture commune à tours les hommes. Sa tâche est des plus édifiantes. Mais elle a besoin d'être épaulée par le juriste. Jentends, par le jeune juriste, car c'est lui seul qui n'est pas entraîné par le mouvement d'horlogerie provenant de l'habitude de penser et d'agir toujours de la mâme manière qui lie l'homme adulte. Le jeune est créateur. Il est pour vivre dans la sérénité. Il porte l'idée et le credo d'une société humaine mondiale.

10.- C'est donc le jeune juriste qui peut servir le mieux $l a$ cause de la paix. Il suffit de réunir les jeunes juristes de différents pays au sein d'organisations internationales pour obtenir des résultats positifs. C'est dans les rencontres de rally des boys scouts ou des camps de la jeunesse et dans les sections de jeunesse de la Croix Rouge ou du Croissant Rouge que nous constatons cette entente et cette harmonie presque incroyable. Deux facteurs sont à considérer: ceux qui se rassemblent sont des jeunes, et les idées qui les rassemblent sont les leurs. Les fondations sont réussies quand ces deux facteurs sont réunis. Des organisations telles que les associations des droits de l'homme ou les associations d'anci- 
ens combattants réussissent parce qu'elles rassemblent les jeut,es ou parce qu'elles persent jeunes.

11.- Ses connaissances juridiques, sa foi en la primauté du droit serviront à donner un élan nouveau aux organisations de niveau ou de caractère international. Car, le jeune juriste, dans le sens que nous venons de définir au paragraphe précédent, agit ainsi que les vases communiquants. En contre partie de ce qu'il apporte à l'organisation internationale, il acquiert et rapporte en son pays d'origine les idées échangées au sein de cet organisme. L'organisation internationale, devient ainsi le lien d'élaboration de la pensée humanitaire. Si des principes tels que la primauté du droit servait de constante pour les réflexions, l'évolution se ferait plus saine.

12.- En réalité, il s'agira d'édifier la conscience collective. La règle "pacta sunt servanda» qui existe depuis des siècles n'a pas empêché les tueries de se produire. Le nouvel essor des droits de t'homme servira sans nul doute à assurer le respect de l'homme à soi-même ct à autrui. La conscience humaine est en train de se faire.

13.- Le noeud ou plutôt le point de départ du problème, réside dans la formation du juriste, c'est-à-dire dans l'enseignement du droit. Le juriste sera ce que vous allez former. Le Professeur François RIGAUX, dans son rapport, donne très savamment et de façon la plus ingénieuse les formules qui serviront à un meilleur enseignement du droit international.

14.- Ce que le jeune juriste doit porter en lui, c'est surtout la conviction en la défense et en la protection des droits de l'hom. me. Rien qu'en se constituant en partisan dévoué et défenseur convaincu des droits de l'homme, il servirait la cause des organisations internationales. La meilleure façon d'éradication de fléaux comme l'apartheid ou la traite des blanches, c'est de créer des occasions permettant aux jeunes juristes de se retrouver dans des rencontres de caractère international. Le fait même qu'ils se réunissent montre leur attachernent à la cause de la paix. Leur échange d'idées fera le reste.

15.- Presque la plupart des organisations internationales ont pour caractère d'être structuré d'après des recettes habituel. les. Leur mécanisme influe sur leur fonctionnement et sur les tâches qu'elles se proposent de réaliser. Elles s'efforcent de rem- 
plir leurs fonctions selon des règles juridiques plutôt surannées. Les jeunes juristes, eux, apportent des cadres nouveaux, des conceptions nouvelles. Ce qui est le plus remarquable, c'est leur comportement auprès des organisations internationales. Ils discutent à coeur ouvert, ils sont sincères, ils n.ont pas d'arrière-pensée, ils sont prèts à faire cause commune pour le bienfait général. Ils sont convaincus que la paix est l'état normal des sociétés humaines, puisque pour eux la valeur suprême réside en l'être humain et que tout doit être jugé en fonction de lui.

16. - C'est dans cet état d'esprit que les jeunes juristes abordent les problèmes. Ils sont les représentants d'une ère nouvelle. Ils sont l'homme de l'ère des droits de l'homme. Et c'est pour cela que les organisations internationales se trouvent dotées d'une âme nouvelle depuis que les jeunes juristes y ont trouvé accès. 